\title{
Sex-Specificity in the Reward Value of Facial Attractiveness
}

\author{
Amanda C Hahn ${ }^{1}$, Lisa M DeBruine ${ }^{1}$, Benedict C Jones ${ }^{1}$
}

${ }^{1}$ Institute of Neuroscience and Psychology, University of Glasgow, UK.

\section{Corresponding author}

Dr Amanda C Hahn

amanda.hahn@glasgow.ac.uk

Institute of Neuroscience and Psychology,

University of Glasgow,

Scotland, UK 


\begin{abstract}
Studies of the sex-specificity of sexual arousal in adults (i.e., the tendency to respond more strongly to preferred-sex individuals than non-preferred sex individuals) have suggested that heterosexual men, homosexual men, and homosexual women show stronger sex-specific responses than do heterosexual women. Evidence for a similar pattern of results in studies investigating the reward value of faces is equivocal.

Consequently, we investigated the effects of (1) sexual orientation (homosexual versus heterosexual), (2) sex (male versus female), (3) image sex (preferred-sex versus nonpreferred-sex), and the (4) physical attractiveness of the individual shown in the image on the reward value of faces. Participants were 130 heterosexual men, 130 homosexual men, 130 heterosexual women, and 130 homosexual women. The reward value of faces was assessed using a standard key-press task. Multilevel modeling of responses indicated that images of preferred-sex individuals were more rewarding than images of non-preferredsex individuals and that this preferred-sex bias was particularly pronounced when more physically attractive faces were presented. These effects were not qualified by interactions involving either the sexual orientation or the sex of our participants, however, suggesting that the preferred-sex bias in the reward value of faces is similar in heterosexual men, homosexual men, heterosexual women, and homosexual women.
\end{abstract}

Keywords: sexual orientation; facial attractiveness; sexual arousal; gender differences; genital arousal 


\section{INTRODUCTION}

In the laboratory setting, the sex-specificity of genital arousal when viewing solitary men and women (i.e., the tendency for greater arousal to occur when viewing preferred-sex individuals than non-preferred-sex individuals) is generally stronger among heterosexual men, homosexual men, and homosexual women than it is among heterosexual women (for a meta-analytic review, see Chivers, Seto, Lalumière, Laan, \& Grimbos, 2010). Weaker specificity in heterosexual women's responses to images of solitary men and women than is seen in heterosexual men's, homosexual men's, or homosexual women's responses has also been reported in studies investigating implicit sexual associations (Snowden \& Gray, 2013), pupil responses to (Rieger \& SavinWilliams, 2012), memory for the faces of (Steffens, Landmann, \& Mecklenbrauker, 2013), neurobiological measures of the reward value of (Kranz \& Ishai, 2006; Sylva et al., 2013), and viewing times for (Ebsworth \& Lalumiere, 2012; Lippa, 2012) preferredsex and non-preferred-sex images. In some of these studies, homosexual women also showed weaker specificity than heterosexual men and homosexual men (e.g., Lippa, 2012; Ebsworth \& Lalumiere, 2012), although homosexual women still showed greater specificity in their responses than did heterosexual women.

Other studies have used key-press tasks, a standard measure of the reward value of images in which participants can exert effort to increase or decrease image-viewing times (Aharon et al., 2001), to investigate the specificity of heterosexual participants' responses to images of preferred-sex and non-preferred-sex faces. Neuroimaging work has shown that the amount of effort that participants will exert to view a given face on these key-press tasks is a particularly good predictor of the extent to which that face will 
elicit activity in brain regions associated with processing of rewards and motivation (Aharon et al., 2001). While many studies have reported weaker specificity in responses to images of preferred-sex and non-preferred-sex individuals in heterosexual women, evidence for this pattern of results in studies using key-press tasks is more mixed. For example, Levy et al. (2008) found that heterosexual men, but not heterosexual women, exerted more effort to view images of preferred-sex faces than images of non-preferredsex faces. By contrast, Hahn, Xiao, Sprengelmeyer, and Perrett (2013) found that both heterosexual men and women exerted more effort to view images of preferred-sex faces than of non-preferred-sex faces.

Both Levy et al. (2008) and Hahn et al. (2013) also presented evidence that the degree of specificity observed in key-press responses to preferred-sex and non-preferredsex faces can be influenced by the physical attractiveness of the individuals presented. The tendency for heterosexual men in Levy et al.'s study and heterosexual men and heterosexual women in Hahn et al.'s study to exert more effort to view preferred-sex faces than non-preferred-sex faces was greater for images of physically attractive individuals than for images of relatively unattractive individuals. Using a passive viewing time measure, Lippa (2012) also found that sex-specific responses were greater for images of physically attractive individuals than for images of relatively unattractive individuals. While key-press tasks have been used to investigate the effects of image sex and physical attractiveness on heterosexual participants' responses, no previous studies have investigated homosexual participants' responses.

The purpose of the current study was to investigate the effects of participant sexual orientation (homosexual versus heterosexual), participant sex (male versus 
female), image sex (preferred-sex versus non-preferred-sex), and the physical attractiveness of the individual shown in the image on the reward value of faces. Reward value was assessed using a standard key-press task adapted from those used previously to explore the effects of image sex and physical attractiveness in studies of the reward value of faces in heterosexual participants (Aharon et al., 2001; Hahn et al., 2013; Levy et al., 2008).

Given that several lines of evidence suggest that heterosexual women show weaker sex-specific responses to preferred-sex versus non-preferred-sex individuals, we might expect sex-specific responses to faces to be weaker (or even absent) in heterosexual women compared with heterosexual men, homosexual men, or homosexual women. Moreover, this pattern of results may be modulated by the physical attractiveness of the individuals shown (i.e., be particularly pronounced when physically attractive individuals are presented) (Lippa, 2012). In contrast to these predictions, however, research reporting that physical attractiveness and image sex have similar effects on the reward value of face images in heterosexual men and women (Hahn et al., 2013) suggests that the effects of image sex and physical attractiveness may not necessarily be qualified by higher-order interactions involving either participant sex or participant sexual orientation. Thus, our study will clarify the extent to which attractiveness, participant sex, and participant sexual orientation modulate the specificity of the reward value of preferred-sex versus non-preferred-sex faces.

\section{METHOD}

\section{Participants}


A total of 520 individuals (130 heterosexual men, 130 homosexual men, 130 heterosexual women, and 130 homosexual women) participated in the main part of the study (i.e., participated in the "pay-per-view" key-pressing task). The mean age of these participants was 22.9 years $(\mathrm{SD}=5.1)$. The breakdown of ethnicities in our sample was as follows: 359 White, 41 Asian, 15 Black, 87 other, 18 non-disclosed. Sex and sexual orientation were assessed via a questionnaire asking for sex (male or female) and preferred sex for romantic relationships (male, female, either). Only individuals who responded "male" or "female" when asked to indicate their preferred sex for romantic relationships were recruited for the study and only 130 participants were recruited for each combination of sexual orientation and sex.

The study was conducted online. Previous research on behavioral responses to faces has demonstrated that online and laboratory studies show similar patterns of results (e.g., Jones et al., 2007). Participants were recruited by following links to an online study of responses to face images placed on social bookmarking websites, such as stumbleupon.com. The study was run on our lab's website (faceresearch.org).

\section{Procedure}

Each of the 520 participants in the main study completed a "pay-per-view" keypressing task, similar to those used in previous studies to assess the reward value of faces (Aharon et al., 2001; Hahn et al., 2013; Levy et al., 2008). After initiating each trial by pressing the space bar, this task allows participants to control the viewing duration of each image by pressing designated keys on their keyboard. Here, the length of time a given face was displayed for could be increased by alternately pressing the 7 and 8 keys or decreased by alternately pressing the 1 and 2 keys. Each key press increased or 
decreased the viewing duration by $100 \mathrm{~ms}$. The default viewing duration for each image (i.e., the length of time a face remained onscreen if no keys were pressed) was 4 seconds. A timer bar on the left-hand side of each image provided visual feedback about the amount of time for which a face would remain onscreen at any given point in time (i.e., if no more key-pressing took place). Participants were told that the key-press task would last for a total of 3.5 minutes in order to discourage responses aimed at changing the length of engagement with the task. However, in reality, the total length of the key-press task was dependent on participants' responses. All participants key-pressed at least once during the experiment. The mean total number of key presses across all trials was 628.0 $(\mathrm{SD}=407.4)$

Of the 130 participants in each group, 65 saw male faces in the key-press task and 65 saw female faces in the key-press task. In other words, in each group of participants, half of the group were presented images of the sex they reported being attracted to (i.e., they saw "preferred-sex-faces") and half of the group were presented images of the sex they did not report being attracted to (i.e., they saw "non-preferred-sex-faces"). Trial order was fully randomized and the participants were randomly allocated to only one version of the task (i.e., saw either only male faces or only female faces). Prior to beginning the main part of the study, participants completed a brief training task designed to familiarize them with the key-press procedure. Faces were not presented in this training task. The face stimuli presented were full-color face images of 50 white adult men $($ mean age $=24.2$ years, $\mathrm{SD}=4.0)$ and 50 white adult women $($ mean age $=24.3$ years, $\mathrm{SD}=4.0$ ) from a database of commercially available standardized face images that have been used in other recent facial attractiveness studies (Fisher et al., 2014). 
Photographs were taken under standardized photographic conditions and depicted individuals who were posed front on to the camera with neutral expressions and direct gaze. Images were aligned on pupil position and masked so that clothing was not visible.

\section{Measures}

Because the reward value of faces can partly depend on their perceived physical attractiveness (Aharon et al., 2001; Hahn et al., 2013; Levy et al., 2008), we also collected attractiveness ratings of the face images presented in the key-press task from a separate group of participants. Male and female faces were presented in separate blocks of trials, in which trial order was fully randomized, and attractiveness ratings were made using a 1 (much less attractive than average) to 7 (much more attractive than average) scale. Participants were randomly allocated to rate the attractiveness of either male or female faces. The 50 male faces were rated by 15 heterosexual men, 15 homosexual men, 15 heterosexual women, and 15 homosexual women (i.e., 60 raters in total; mean age $=$ 25.1 years, $\mathrm{SD}=5.6$ ). The 50 female faces were rated by different groups of 15 heterosexual men, 15 homosexual men, 15 heterosexual women, and 15 homosexual women (i.e., 60 raters in total; mean age $=24.7$ years, $\mathrm{SD}=5.6$ ). These participants' sex and sexual orientation were assessed in the same way as the participants in the key-press task.

For each group of raters (i.e., heterosexual men, homosexual men, heterosexual women, homosexual women), inter-rater agreement in attractiveness ratings was high for both the male and female faces, all Cronbach's alphas $>.81$. Consequently, we calculated the mean attractiveness rating for each face separately from each groups' ratings. For male faces, these mean ratings were highly and positively intercorrelated among the four 
groups, all $r>.70$, all $\mathrm{N}=50$, all $p<.001$. For female faces, the mean ratings were also highly and positively intercorrelated among the four groups, all $r>.75$, all $\mathrm{N}=50$, all $p<$ .001. Given these intercorrelations, we combined mean ratings to produce a single attractiveness score for each face by collapsing (i.e., averaging) scores across the four rater groups. These combined attractiveness scores were used as the physical attractiveness measure in our analyses.

\section{Statistical Analyses}

We used multilevel analyses with cross-classified structures conducted using R ( R Core Team, 2013), lme4 (Bates, Maechler, Bolker, \& Walker, 2014), and lmerTest (Kuznetsova, Brockhoff, \& Christensen, 2013). Following previous work (e.g., Hahn et al., 2013; Levy et al., 2008), each participant's key-press score for each face image was calculated by subtracting the total number of key presses to decrease a face's viewing duration from the total number of key presses to increase that face's viewing duration. These key-press scores served as the dependent variable in our analyses. Participant sex $(0=$ female, $1=$ male $)$, participant sexual orientation $(0=$ heterosexual, $1=$ homosexual $)$, image sex $(0=$ non-preferred, $1=$ preferred $)$, and physical attractiveness (centered) were included in the models as fixed effects. Participant sex, participant sexual orientation, and image sex were entered at the participant level, while physical attractiveness was entered at the face level. Random effects of participant and face were included in each model.

\section{RESULTS}

In the initial model, we included all possible interactions among the fixed effects. This model showed a significant effect of participant sexual orientation $(t=2.48, p=$ .013), whereby homosexual participants had, on average, greater key-press scores than 
did heterosexual participants. The model also showed a significant effect of image sex $(t$ $=2.16, p=.031$ ), whereby participants had, on average, greater key-press scores for preferred-sex faces than they did for non-preferred-sex faces. A significant positive effect of physical attractiveness $(t=4.58, p<.001)$ indicated that participants had greater keypress scores for more attractive non-preferred-sex faces. The interaction between image sex and physical attractiveness was also significant in this model $(t=4.07, p<.001)$, indicating that the positive effect of physical attractiveness on key-press scores was significantly greater for preferred-sex faces than non-preferred-sex faces. No other effects or interactions were significant (all $t<1.61$, all $p>.10$ ). Together, these results show that participants generally had greater key-press scores for preferred-sex than non-preferredsex faces and that this effect was particularly pronounced for physically attractive faces, but was not modulated by participant sex and/or participant sexual orientation. Figure 1 shows the relationship between facial attractiveness and key-press scores for each combination of participant sex, participant sexual orientation, and image sex.

Next, we repeated the analysis after removing all non-significant interactions. This model also showed the significant effects of participant sexual orientation $(t=3.99$, $p<.001)$, image sex $(t=2.32, p=.021)$, and physical attractiveness $(t=10.33, p<.001)$, as well as the significant interaction between image sex and physical attractiveness $(t=$ $7.73, p<.001)$. The effect of participant sex remained non-significant $(t=-0.43)$.

To test whether the results might be affected by an own-race bias in face processing abilities (see Meissner \& Brigham, 2001 for a meta-analytic review), we repeated both of these models for a dataset consisting of the 359 participants in our study 
who described their ethnicity as white. These models showed the same pattern of significant results as the two models described above.

\section{DISCUSSION}

We examined the effects of participant sexual orientation, participant sex, image sex, and the physical attractiveness of the individual shown in the image on the reward value of faces. Our analyses showed that images of preferred-sex individuals were more rewarding than images of non-preferred-sex individuals. Furthermore, this preferred-sex bias was modulated by the physical attractiveness of the faces shown; the tendency for preferred-sex faces to be more rewarding than non-preferred-sex faces was greater when more physically attractive faces were presented.

Importantly, our analyses also demonstrated that the effects of attractiveness and image sex were not modulated by either the sexual orientation or the sex of our participants. These results suggest that the reward value of faces shows sex specificity (i.e., preferred-sex faces are more rewarding than non-preferred-sex faces, particularly when attractive faces are presented), but that this specificity was similar among homosexual and heterosexual men and women. These results were consistent with some previous work on the reward value of faces that used similar key-press tasks to investigate heterosexual participants' responses to male and female faces (Hahn et al., 2013) and extend that work by showing a similar pattern of responses in homosexual participants. Our results were also consistent with previous work using neurobiological measures of the reward value of faces (Ishai, 2007), which reported similar specificity in the reward value of attractive faces among homosexual and heterosexual men and women. 
As outlined in the Introduction, studies of genital arousal (Chivers et al., 2010), implicit sexual associations (Snowden \& Gray, 2013), pupil responses (Rieger \& SavinWilliams, 2012), and passive viewing times (Ebsworth \& Lalumiere, 2012; Lippa, 2012) have reported greater specificity in responses to images of solitary men and women in heterosexual men, homosexual men, and homosexual women than was observed in heterosexual women's responses. By contrast with these findings, our study of key-press responses found no evidence that participant sex or sexual orientation modulated the reward value of preferred-sex versus non-preferred-sex faces. However, our results are consistent with findings for subjective measures of sexual arousal, in which the extent to which people reported arousal in response to preferred-sex and non-preferred-sex stimuli was similar in men and women regardless of their sexual orientation (Both, Spiering, Everaerd, \& Laan, 2004; Kukkonen, Binik, Amsel, \& Carrier, 2010).

In summary, we found that the reward value of faces was modulated by both image sex (i.e., preferred-sex faces were more rewarding than non-preferred-sex faces) and the physical attractiveness of the individuals presented (i.e., more attractive images were more rewarding). Moreover, there was an interaction between the effects of image sex and attractiveness, whereby the tendency for preferred-sex faces to be more rewarding than non-preferred-sex faces was particularly pronounced when more attractive images were presented. By contrast, none of these effects were modulated by participant sex or participant sexual orientation, suggesting that the sex-specificity of the reward value of faces in general and facial attractiveness specifically is similar in heterosexual men, homosexual men, heterosexual women, and homosexual women. This pattern of results differs from that seen for objective measures of sexual arousal, raising 
the possibility that reward value as measured by responses on a key-press task may reflect factors other than sexual arousal. 


\section{REFERENCES}

Aharon, I., Etcoff, N., Ariely, D., Chabris, C. F., O’Connor, E., \& Breiter, H. C. (2001). Beautiful faces have variable reward value: fMRI and behavioral evidence. Neuron, $32,537-551$.

Bates, D., Maechler, M., Bolker, B., \& Walker, S. (2014). lme4: Linear mixed-effects models using Eigen and S4. R package version 1.0-6. http://CRAN.Rproject.org/package $=1 \mathrm{me} 4$

Both, S., Spiering, M., Everaerd, W., \& Laan, E. (2004). Sexual behavior and responsiveness to sexual stimuli following laboratory-induced sexual arousal. The Journal of Sex Research, 41, 242-258.

Chivers, M. L., Seto, M. C., Lalumière, M. L., Laan, E., \& Grimbos, T. (2010). Agreement of self-reported and genital measures of sexual arousal in men and women: a meta-analysis. Archives of Sexual Behavior, 39, 5-56.

Ebsworth, M., \& Lalumiere, M. L. (2012). Viewing time as a measure of bisexual sexual interest. Archives of Sexual Behavior, 41, 161-172.

Fisher, C., Fincher, C. L., Hahn, A. C., Little, A. C., DeBruine, L. M., \& Jones, B. C. (2014). Do assortative preferences contribute to assortative mating for adiposity? British Journal of Psychology, 105, 474-485. doi: 10.1111/bjop.12055

Hahn, A. C., Xiao, D. K., Sprengelmeyer, R. H., \& Perrett, D. I. (2013). Gender differences in the incentive salience of infants. Quarterly Journal of Experimental Psychology, 66, 200-208.

Ishai, A. (2007). Sex, beauty and the orbitofrontal cortex. International Journal of Psychophysiology, 63, 181-185. 
Jones, B. C., DeBruine, L. M., Little, A. C., Conway, C. A., Welling, L. L. M., \& Smith, F. G. (2007). Sensation seeking and men's face preferences. Evolution and Human Behavior, 28, 439-446.

Kranz, F., \& Ishai, A. (2006). Face perception is modulated by sexual preference. Current Biology, 16, 63-68.

Kukkonen, T. M., Binik, Y. M., Amsel, R., \& Carrier, S. (2010). An evaluation of the validity of thermography as a physiological measure of sexual arousal in a nonuniversity adult sample. Archives of Sexual Behavior, 39, 861-873.

Kuznetsova, A., Brockhoff, P. B., \& Christensen, R. H. B. (2013). lmerTest: Tests for random and fixed effects for linear mixed effect models (lmer objects of lme4 package). $R$ package version 2.0-3. http://CRAN.R-project.org/package=lmerTest

Levy, B., Ariely, D., Mazar, N., Chi, W., Lukas, S. E., \& Elman, I. (2008). Gender differences in the motivational processing of facial beauty. Learning and Motivation, 39, 136-145.

Lippa, R. (2012). Effects of sex and sexual orientation on self-reported attraction and viewing times to images of men and women: Testing for category specificity. Archives of Sexual Behavior, 41, 149-160.

Meissner, C. A. \& Brigham, J. C. (2001). Thirty years of investigating the own-race bias in memory for faces: A meta-analytic review. Psychology, Public Policy, and Law, 7, 3-35.

R Core Team. (2013). R: A language and environment for statistical computing. $R$ Foundation for Statistical Computing, Vienna, Austria. URL http://www.Rproject.org/. 
Rieger, G., \& Savin-Williams, R. C. (2012). The eyes have it: Sex and sexual orientation differences in pupil dilation patterns. PloS ONE, 7, e40256.

Snowden, R., \& Gray, N. S. (2013). Implicit sexual associations in heterosexual and homosexual men and women. Archives of Sexual Behavior, 42, 475-485.

Steffens, M. C., Landmann, S., \& Mecklenbrauker, S. (2013). Participant sexual orientation matters: New evidence on the gender bias in face recognition. Experimental Psychology, 60, 362-367. 


\section{Acknowledgements}

Parts of this research were supported by European Research Council Starting Grant 282655 (OCMATE), awarded to Benedict C. Jones. 

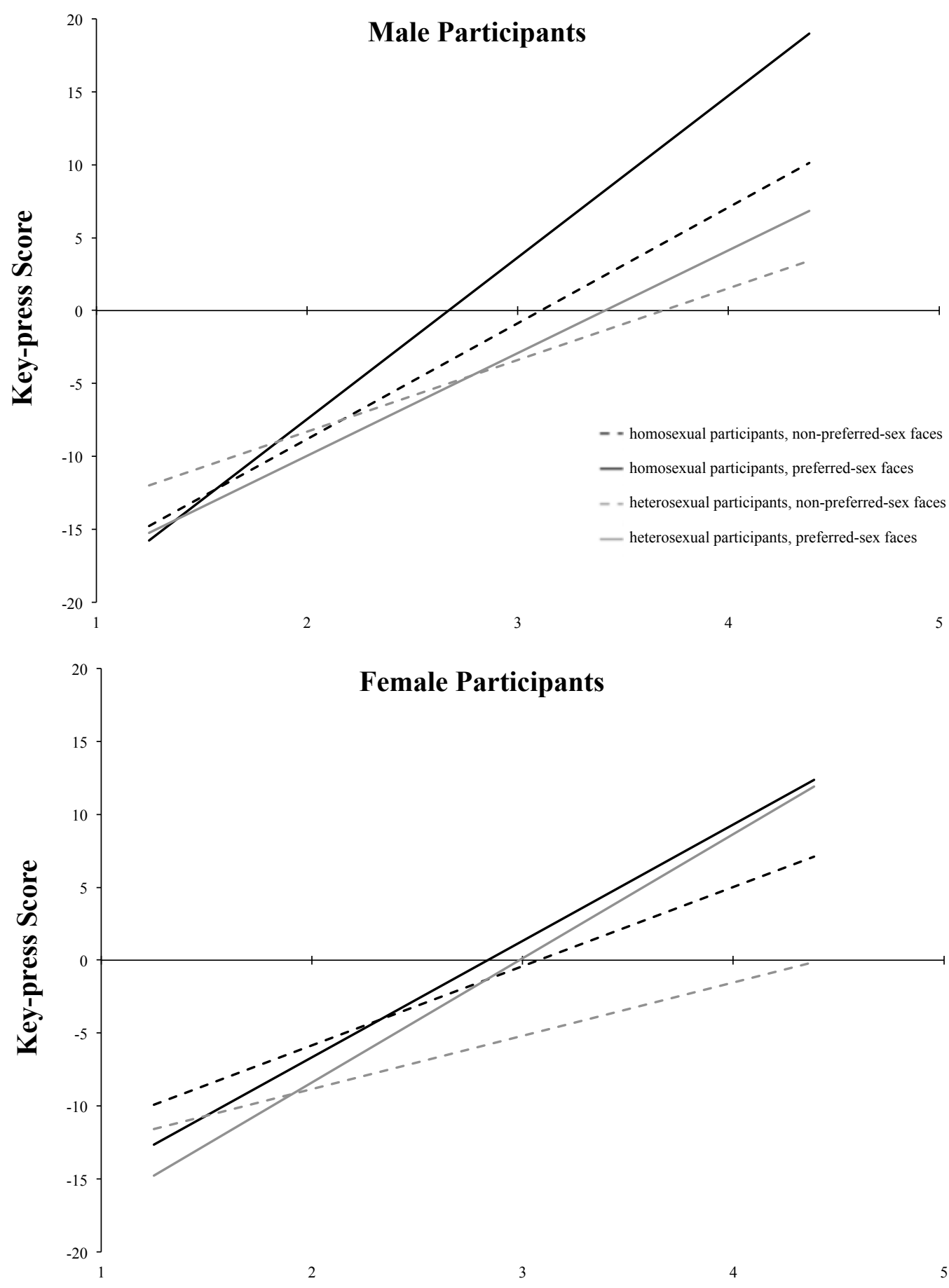

Facial Attractiveness

Figure 1. Correlations between key-press scores and third-party ratings of facial attractiveness for preferred-sex and non-preferred-sex faces. The top panel shows male participants' data and the bottom panel shows female participants' data. 